\title{
Development of preparatory activity indexed by the Contingent Negative Variation in Children
}

Angélica B. Flores, Marcia R. Digiacomo, Susana Menares, Eva Trigo and Carlos M. Gómez

Address: Departamento de Psicología Experimental, Facultad de Psicología. Universidad de Sevilla, c/Camilo José Cela s/n, Sevilla 41018, Spain.

Email: Angélica B. Flores - angelicafloresb@gmail.com; Marcia R. Digiacomo marciadigi@hotmail.com, Susana - susana@fundacionicse.com, Eva Trigo - trigo@us.es; Carlos M. Gómez - cgomez@us.es

Corresponding author:

Angélica B. Flores. Departamento de Psicología Experimental, Facultad de Psicología. Universidad de Sevilla, angelicafloresb@gmail.com 


\begin{abstract}
Objectives: The present study investigated the influence of the cerebral maturity in the process of cue task-specific preparatory activation induced by an spatial cue using a central cue Posner paradigm. For these purposes, the behavioral responses and the Contingent Negative Variation (CNV) generated between the S1 (the warning stimulus) and the S2 (the imperative stimulus) were compared in 16 healthy children (8-13 years old) and 17 healthy young adults (18-23 years old).

Methods: The EEG was recorded from 20 scalp sites of the International 10-20 system. The complete trial period included a central directional cue that was on for $300 \mathrm{~ms}$ and an attentive waiting period lasting $1360 \mathrm{~ms}$. Finally, a peripheral target subtending a visual angle of $4.56^{\circ}$ and situated $2.28^{\circ}$ eccentrically in the horizontal meridian appeared. The early and late components of the CNV appearing in the period between the cue and the target were analyzed.

Results: The CNV of children did not show contralateral cortical activation related to motor preparation. However, the young adults showed contralateral to the cue activation in motor areas. The children and young adults showed cortical activation in the posterior sensory areas, displaying a contralateral to the cue pattern of activation. In addition to this, a parietal positive component appeared in children during the CNV period.

Conclusions: These results might reflect a higher immaturity of the motor preparation system compared with the sensory preparatory system in children. It is possible that children used different strategies than young adults to prepare for stimuli and responses.
\end{abstract}

Key words: Contingent Negative Variation; child development; Posner paradigm; anticipatory attention; motor preparation; sensory preparation; slow brain potentials;

\title{
I. Introduction
}


The motor and sensory preparation can be considered an executive function that wrap on the psychophysiological processes. With Event Related Potentials (ERPs), it is possible to study the preparation of specific cerebral areas to execute a task, the evaluation of the incoming stimuli for the correct execution of the task and the influence of the cerebral maturation in these processes.

The preparation for a motor response has been studied with the Contingent Negative Variation $(\mathrm{CNV})$. This slow negative (ERP) is usually obtained during preparatory periods for perception and action. The classic paradigm to obtain the CNV uses the presentation of a warning stimulus (S1) and, after a short interval of time (preparation), an imperative stimulus (S2) (Walter et al., 1964). The CNV is a complex process with two subcomponents described. The initial component reflects orientation to the warning stimulus (early CNV) (Weerts and Lang, 1973), whereas the later component indexes anticipation of the imperative stimulus (Weerts and Lang, 1973), including motor preparation for the response (late CNV) (Loveless and Sanford, 1974), sensory anticipation (Brunia et al., 1999, Gómez et al., 2003 and 2004) and the activation of the attentionally-related fronto-parietal networks (Gómez et al., 2007, Fan et al., 2007).

The aim of preparatory processes would be to pre-activate certain brain structures during that interval in order to improve the information processing of the upcoming imperative stimulus. The CNV would reflect pre-activation of the sensory areas which would process the incoming stimulus and the motor areas needed to execute the motor programs upon the information conveyed by the S1 (Brunia, 1999, Brunia and Van Boxtel, 2001, Gomez, et al., 2003, 2004). Several studies have suggested that the premotor area and the supplementary motor area are involved in the preparation for the motor task (Brunia and Damen, 1988; Lee et al. 1999; Gómez et al., 2004). The activation of primary and secondary sensory areas has also been proposed for the sensory preactivation in the post-warning period (Gómez et al., 2001, 2003 and 2004). This task-specific preparatory activity would be particularly clear in central cue-Posner paradigms, in wich a central cue indicates with a certain probability the location where it would appear the target. This sensory and motor orientation to the cue produces a pattern of cost-benefit (Posner, 1980), also called 
validity-invalidity effect. It has been proposed that the task-specific network prepared during the S1-S2 period becomes efficient or inefficient and requires an attentional re-orientation as a consequence of the correct or incorrect prediction of the target (Gómez et al., 2004).

This preparation has consequences on the brain processing of the upcoming target. The early post-target ERPs have shown to be modulated when the target is preceded by an spatial cue, in adults (Mangun and Hillyard, 1991) and in children (Perchet and García-Larrea, 2000, 2005). Moreover, an increase of the posterior positive components for invalid condition with respect to valid conditions at the time latency of the P3 component has also been described (Mangun and, Hillyard, 1991; Eimer, 1993). Recently, (Gómez et al., 2008) the P3b effects have been replicated, but an increase of the P3a to invalidly cued targets have also been observed.

There are few studies about the CNV developmental course during central cue Posner paradigms. Perchet and Garcia-Larrea $(2000,2005)$ have assessed the attention shifting and response selection tasks in spatially cued motor reaction task in the 'Posner' paradigm in children and adults. In adults, they reported slow negative potential preceding the target in the valid, invalid, and no cue experimental conditions; whereas in children, this potential does not appear in valid and invalid condition, only during the 'no cue', these differences were interpreted as a lack of maturation in executive process (Perchet and Garcia-Larrea, 2005). However, they used a very short interval between the cue and the target $(500 \mathrm{~ms})$ to allow a fully developed CNV.

Other studies provided information about brain activity in normal children during preparatory periods by means of the CNV. Jokman et al. (2003) performed a go-nogo task and compared children (9-10 years old) and adults (20 years old). In the early CNV in centro-parietal locations, the CNV amplitude was smaller in children than in adults. This activity was associated with inefficient cue-orientation processes caused by incomplete frontal lobe development. Segalowitz and Davies (2004) compared children (7-17 years old) and adults (19-25 years old). These authors, taking into account other ERPs as the error related negativity and the novelty P3a, proposed that the frontal lobe continues developing during adolescence, in parallel with the 
growing amplitude of CNV with age, associated with the maturation of the frontal lobe generators. Another investigation (Jokman, 2006) studying two groups of children (6-7 and 9-10 years old) and young adults (19-23 years old) reported that the early CNV amplitude was reduced in the younger children. This result was interpreted as an immature fronto-parietal networks in children that are involved in motor regulation. Other authors reported increasing CNV amplitudes with increasing age (Cohen, 1970; Cohen et al., 1967; Bender, 2002, Segalowitz et al., 1992).

On the other hand, the studies about the cortical CNV generators of the motor component during childhood and pre-adolescents showed that the motor system has a late maturation in young children with respect to adolescents and young adults (Bender et al., 2002). In another report, Bender et al. (2005) established differences in the CNV between young children and adolescents. The major differences arose from the absence of premotor $\mathrm{CNV}$ in young children (6-11 years old) with respect to adolescents (12-18 years old). Previous developmental studies have shown that the Bereitschaftspotential potential (BP), which is a negative potential in adults indexing motor preparation, inverts its polarity from positive to negative in children aging from 6 to 8 years old (Chiarenza et al., 1983). Bender et al. (2005) also showed that the auditory sensory component of the $\mathrm{CNV}$ would develop earlier than the motor component. Other reports involve a comparison between clinical and control groups (Yordanova, et, al, 1997, Perchet, 2001). A reduced early or frontal CNV activity has been reported in ADHD children (Yordanova, et al, 1997).

The general objective of the present report is to establish the influence of the cerebral maturity in the preparation of task-specific sensory and motor cerebral areas induced by a spatial central cue. The specific objectives are studying the influence of the cerebral maturity in: (i) the orientation process to warning stimuli by means of the early component of the CNV, (ii) the preparatory activation of the sensory and motor areas upon the information conveyed by the S1, by means of the late component of the CNV. It is possible that the development of preparation of the orientation, the sensory and the motor subcomponents would be influenced by the differential maturation of the cortical regions. 


\section{Method}

\subsection{Participants}

Forty healthy subjects participated in this experiment: 20 children (12 female and 8 male, 18 right-handed and 2 left-handed) between 8 and 13 years old (mean age: $10.05-+1.5$ S.D.) and 20 young adults (12 female and 8 male, all right-handed) between 18 and 23 years old (mean age: $20.56+-1.3$ S.D.). The subjects with high eye movements were discarded for the analysis (see below). This reduced the sample to 16 children (11 female and 5 male, 15 right-handed and 1 left-handed) between 8 and 13 years old (mean age: 10.13 -+1.4 S.D.) and 17 young adults (9 female and 8 male, all right-handed) between 18 and 23 years old (mean age: $20.56+-1.3$ S.D). The parents of the children and the young adults were informed about the general characteristics of the experiment and they gave an informed consent prior to the start of the study. The experiments were conducted following the rules of the Helsinki Convention.

\subsection{Stimuli and behavioural paradigm}

The stimulus presentation was computer-controlled (EEVOKE, ANT). Participants were seated 50 $\mathrm{cm}$ in front of a computer screen. They were instructed to settle their eyes on a white square in the centre of the screen. The white spot was on during the whole experiment in order to keep the central fixation. The complete trial period included a central directional cue that was on $300 \mathrm{~ms}$, and then, an attentive wait period lasting $1360 \mathrm{~ms}$. Finally, a peripheral target subtending a visual angle of $4.56^{\circ}$ and situated $2.28^{\circ}$ eccentrically in the horizontal meridian appeared (Fig. 1). The targets were cartoon figures that were constant in each block of trials. As there were 5 blocks of trials, 5 different figures were used in the whole experiment. The directional central cue could indicate the right or left direction in which the target would appear: validly cued targets (82.1\% of trials) (VC) and invalidly cued targets (IC) (17.9\% of trials). The subjects used the right index finger to respond to the right targets by pressing a joystick button, and correspondingly they used the left index finger to respond to the left 
targets. The inter-trial intervals had a duration of $1860 \mathrm{~ms}$. Subjects were presented with a total of 235 trials. The task was practiced with some training trials (10 trials) before beginning the experimental session.

\subsection{Electrophysiological recording}

The EEG was recorded from 20 scalp sites of the International 10-20 system (Fp1, Fp2, F3, F4, F7, F8, Fz, FCz, T7, T8, C3, C4, Cz, P7, P8, P3, P4, Pz, O1, O2) using tin electrodes mounted in an electrode cap (EASYCAP, Herrsching-Breitbrunn, Germany). Two additional electrodes (M1, M2) were recorded on the left and right mastoid. Horizontal eye movements were recorded from two electrodes at the outer canthus of each eye, and one electrode under the left eye compared to Fp1 was used for recording vertical eye movements. Impedances were maintained below $10 \mathrm{ohms}$. They were acquired at a sampling rate of $512 \mathrm{~Hz}$, using a commercial AD acquisition and analysis board (ANT) and the amplification gain was 20,000 (ANT amplifiers).

\subsection{Data and statistical analysis}

Behavioural measures. The RTs were measured in the valid and invalid trials. The RTs were analyzed with mixed-design ANOVA with types of trials (valid cue and invalid cue) as withinsubjects and groups (children and young adults) as between-subjects factor.

The percentages of errors were computed in valid and invalidly cued trials: the percentage of no response (Omissions to the S2), percentage of false alarms to the S1 (Responses to the S1) and percentage of commission errors to the target stimuli (Response errors to the S2) (Table 2). The omissions to the S2 and response errors to the S2 were analyzed with mixed-design ANOVA with types of trials (valid cue and invalid cue) as within-subjects and groups (children and young adults) as between-subjects factor. The responses to the $\mathrm{S} 1$ were not separated in invalid and valid conditions. These errors were analyzed with one-way ANOVA for comparing children with young adults. 
Event-related potential. The average reference that was used for recording the EEG was changed to an algebraically linked mastoids (M1 + M2/2) for the ERP analysis. Vertical EOG artefacts were removed from the data by applying an eye-movement correction with the SOBI algorithm (Software ANT, version 4.1.1.). The EEG epochs that after the application of the SOBI algorithm exceeded 100 microvolts in any channel were automatically discarded. Subjects that once computed the artefact rejection presented a systematic eye derive higher than +- 10 microvolts in young adults, and +-20 microvolts in children were discarded for further analysis. These voltage values correspond in adults to a visual angle maximum of 1 degree, and there is no reference in the literature for children (Hillyard and Galambos, 1970). This procedure resulted in discarding 4 subjects.

\subsubsection{Contingent Negative Variation (CNV)}

The CNV amplitude in response to cues of children and young adults was compared in order to assess possible differences in preparatory processes between children and young adults. EEG epochs were obtained separately for right and left cue trials. The EEG was segmented into epochs of $2300 \mathrm{~ms}$, with a baseline from 300 to $0 \mathrm{~ms}$ preceding the stimulus (S1). Therefore, the post-stimulus period was $2000 \mathrm{~ms}$. Average amplitude was measured in three different time windows post-cue, the first window was different in each group because the negative waves appeared earlier in children than in young adults: $330-450 \mathrm{~ms}$ (children) and 450-550 ms (young adults), the second window was 550-950 ms and the third window was $1560-1660 \mathrm{~ms}$ for both children and young adults. The scalp was divided in 9 regions: left-frontal (collapsing Fp1 and F3 electrodes), midfrontal (Fz), right-frontal (Fp2, F4), left-central (C3), midcentral (Fcz, Cz), right-central $(\mathrm{C} 4)$, left-posterior (P3, $\mathrm{P} 7, \mathrm{O} 1)$, midposterior $(\mathrm{Pz})$ and right-posterior area $(\mathrm{P} 4, \mathrm{P} 8, \mathrm{O} 2)$. Different subregions were analyzed for the analysis of different time windows.

The Mixed-Model Factorial ANOVA for the early time window of the CNV (children: 330$450 \mathrm{~ms}$ and young adults: $450-550 \mathrm{~ms}$ ) was not computed because the children showed a frontocentral negativity earlier than the young adults, but also because the children showed a positivity 
posterior which coincided in latency with the fronto-central negativity (Fig.2). The early positivity and negativity in children are possibly a manifestation of the same component but with an opposite polarity. Therefore, the fronto-central negativity would not correspond to the early component of the CNV (see correlational and principal component analysis to support this hypothesis). A correlational analysis of the amplitude voltage of posterior vs. anterior areas was computed. Additionally, the principal component analysis (PCA) using the BESA software was applied to the data. Both analysis were performed in order to establish if the fronto-central and posterior waves represent the same or different sources of variance.

Therefore, for the analysis of the early $\mathrm{CNV}$ in children, a factorial repeated measure ANOVA with two factors was computed: cue (left and right) and scalp area (left-frontal, midfrontal, right-frontal, left-central, midcentral, right-central). For the posterior positivity, the same kind of ANOVA was computed but changing the areas to posterior areas: left-posterior, midposterior and right-posterior.

The Early CNV (450-550 ms) in young adults was compared with the linebase (300 ms prior to S1) in order to check that the small appreciated negativity was over noise level and could be considered a genuine early CNV component. Therefore, a factorial repeated measure ANOVA was performed with two factors: latency (early CNV and baseline) and scalp areas (left-frontal, midfrontal, right-frontal, left-central, midcentral and right-central areas).

For the analysis of the middle (550-950ms) and late latency (1560-1660 ms) of the CNV, a mixed-model factorial ANOVA with cue (left and right) and scalp areas as within-subjects factors and age group (children and young adults) as between-subjects factor was computed. This approach would allow not only to assess differences due to the age, but also if there is a differential activation of the different brain areas as a consequence of the cued spatial region. Particularly, the analysis would allow to establish if there is a contralateral to the cued side motor and sensory preparation. To specifically assess the sensory preparation, the analyzed areas were the posterior occipitoparietal (left-posterior, mid-posterior and right-posterior) areas, which are the scalp areas related to 
striate and extra-striate cortex. Moreover, another similar Mixed-model factorial ANOVA with cue direction (left and right) and scalp areas (left cue $\mathrm{C} 3$, left cue $\mathrm{C} 4$, right cue $\mathrm{C} 4$, right cue $\mathrm{C} 3$ ) as within-subjects factors and group (children and young adults) as between-subjects factor was applied to assess motor functions. The scalp areas were $\mathrm{C} 3$ and $\mathrm{C} 4$ in order to show the possible preparatory activation of left or right motor areas.

For all analyses a level of significance $p=.05$ was adopted as a statistically significant level. The Greenhouse-Geisser correction was applied when it was necessary. The test $F$ Welch was applied instead of the $F$ Snedecor when the variance of both groups was different. The Bonferroni method was used as a post hoc test when it was necessary.

\section{RESULTS}

Behavioral measures. The RTs were analyzed with a mixed-design factorial ANOVA with types of trials (valid cue and invalid cue) as within-subjects and groups (children and young adults) as between-subjects factor. The RTs of valid and invalid trials appeared in Table 1. The mixeddesign ANOVA showed statistically significant differences of the effect of the types of trial, $F$ (1, $31)=15.95, p=.000$. The valid condition $(M=368.36, S D=84.13)$ was faster than invalid condition $(M=405.25, S D=82.49)$. There were not statistically significant differences among age groups, $F(1,31)=2.59, p=0.118)$, although the averages showed that children RTs were somehow higher than those of young adults (Table 1). There was not statistically significant interaction between the effects of types of trial and groups, $F(1,31)=.61, p=.441$.

The percentages of errors in the valid and invalid condition are presented in Table 2. The omissions to the S2 analyzed with Mixed-design ANOVA with types of trial (valid cue and invalid cue) and groups (children and young adults) as factors showed statistically significant differences of the effect of types of trial, $F(1,31)=4.676, p=.038$. The invalid condition presented higher number of omission errors $(M=8.99, S D=6.56)$ than the valid condition $(M=6.64, S D=4.55)$. There were not significant differences between groups. However, the children presented a higher 
number of omission errors than young adults in both conditions (Table 2). There was not statistically significant interaction of the effects of types of trial and groups. The response errors to the S2 (commission errors) analyzed with mixed-design ANOVA with types of trial (valid cue and invalid cue) and groups (children and young adults) showed statistically significant differences of the effect of the types of trial, $F(1,31)=14.57, p=0.001$. The invalid condition presented a higher number of commission errors $(M=3.97, S D=5.08)$ than the valid condition $(M=.96, S D=1.55)$. Welch's correction for heterogeneity of variances showed significant differences between groups $F(1,24.66)=6.33, p=.020$. The children $(M=3.71, S D=3.34)$ presented a higher number of commission errors than young adults $(M=1.30, S D=2.05)$. There was not significant interaction of the effects of types of trial and groups. The responses to the S1 analyzed with one-way ANOVA showed statistically significant differences between groups $F$ de Welch $(1,23.068)=9.88, p=.004$. The children $(M=1.08, S D=.76)$ presented a higher number of responses to $\mathrm{S} 1$ errors than the young adults $(M=.42, S D=.42)$.

\section{Event-related potentials (ERPs).}

\subsection{Contingent Negative Variation.}

Both age groups displayed an early fronto-central negativity, but in different time windows (see Figure 2). In the first analyzed time window (children in $330-450 \mathrm{~ms}$ and young adults in 450$550 \mathrm{~ms}$ ), the age groups were analyzed in a separated manner (see the methods section and below). Some remaining horizontal eye movements were still present when the cue pointed to the right (see figures 2 and 3) (see below to discard influence on the obtained CNV results).

For the children, the repeated measures ANOVA on the frontal negativity showed a significant effect of the scalp area factor $F(2.00,37.44)=9.69, p=0.001$ (Greenhouse-Geisser corrected). There were no other statistically significant effects. For the posterior area, the ANOVA showed a significant effect of the scalp area factor $F(1.31,19.64)=16.74, p=.001$ (GreenhouseGeisser corrected). No other statistically significant effects were observed. In both the anterior 
negativity and the posterior positivity, there were not statistically significant differences due to the cue factor. For this reason, the ERPs and the maps presented in Figure 2 are collapsed for the left and right cue stimulation.

As the anterior negativity and the posterior positivity presented similar time dynamics but with opposite polarities (see Figure 2), a correlational analysis between anterior and posterior areas was performed. The correlation analysis between fronto-central areas and posterior areas showed significant negative Pearson correlations between left-posterior area and left-frontal area $(r=-.565$, $p=.023)$, left-posterior area and midfrontal area $(r=-.682, p=.004)$, left-posterior area and rightfrontal area $(r=-.568, p=.022)$, right-posterior area and left-frontal area $(r=-.539, p=.031)$, right-posterior area and midfrontal area $(r=-.721, p=.002)$, right-posterior area and right-frontal area $(r=-.527, p=.036)$ and significant positivity correlation between midposterior area and rightcentral area $(r=.784, p=.000)$. The negative correlation between posterior and anterior sites indicates that the posterior positivity and the fronto-central negativity could arise from the same source of variance. The PCA analysis in the first time window in children showed that the variance explained by the first spatial component was $98.8 \%$. Moreover, the topography of the first spatial component showed that the recorded anterior negativity and posterior positivity would be manifestations of the same component but with opposite polarities in anterior and posterior sites (see Figure 2). Also observe that the topography of the first spatial component is completely similar to that of the fronto-central negativity in children.

The comparison in the fronto-central areas between early CNV and the baseline amplitude in young adults showed statistically significant values $F(1,16)=11.84, p=.003$, indicating that the small fronto-central negativity in young adults is, in fact, a genuine early component of the CNV (Fig. 2). The early CNV amplitude $(M=-1.59, S D=.50)$ presented a higher amplitude than the young adults baseline.There were not other statistically significant effects.

In the time window of 550-950 ms, the mixed-design ANOVA analysis with cue (left and right) and posterior areas (left-posterior, midcentral-posterior, right-posterior areas) as within- 
subjects factors and age group (children and young adults) as between-subjects factor showed statistically significant effects of the interaction between the effects of the age groups and scalp areas $F(1.72,53.28)=8.32, p=.001$ (Greenhouse-Geisser corrected). The figures 3 and 4 a showed that the children have a more positive value than the young adults in the midposterior area. In order to know which posterior areas presented different voltage in children and young adults, we analyzed the three simple main effects of groups with Bonferroni correction $\left(\alpha_{\mathrm{PC}}=.05 / 3=.016\right)$. Maximum difference between groups was observed in midposterior area, and showed a trend to statistical significance, $F(1,31)=5.82, p=.022$. Moreover, the mixed-design ANOVA analysis also showed statistically significant interaction between the effects of the cue and posterior areas factors $($ Geenhouse-Geisser $F(1.90,58.79)=13.65, p=.001)$. The figure $4 \mathrm{~b}$ shows this interaction: when the cue indicates toward the right, the left-posterior area becomes the most negative area, and when the cue indicates toward the left, the right-posterior area becomes the most negative area (Figure 3). These results indicate that the posterior areas contralateral to the cued direction became preactivated during this intermediate $\mathrm{CNV}$ period for both the children and young adults. In order to know if right and left posterior areas presented different amplitudes when left cues and right cues were presented, the bonferroni test was applied $\left(\alpha_{\mathrm{PC}}=.05 / 3=.016\right)$. A trend to statistically significant differences was observed between right cue and left cue in right-posterior areas, $(p$ $=.022)$, and between right cue and left cue in left-posterior areas $(p=.069)$. There were not significant differences between age groups and the effects of the interaction between types of cue, scalp area, and age groups. These statistical trends allowed us to test the hypothesis of a contralateral to the cue negative activation in posterior sites by means of a t-test. For that, left and right cue conditions were collapsed, and the amplitude values of contralateral and ipsilateral to the cue CNV values in posterior sites were compared. The t-test showed a statistically significant negativity in posterior sites contralateral to the cue when compared to homologous positions in the ipsilateral to the cue side $(\mathrm{t}=-3.58 ; \mathrm{p}=0.001)$. 
The possible activation of motor areas was also analyzed. The mixed-model ANOVA with cue, scalp area and group showed that the effect of the group was statistically significant using the $F$ de Welch $(1,23.29)=5.30, p=.028$. There were not more significant effects in this analysis. Additionally, we analyzed independently each group of age with a repeated measures ANOVA analyses with cue direction (left and right) and scalp area (C3, C4) as factors. The young adults showed a clear trend to statistically significant differences in the interaction between the effects of the cue direction and the scalp area, $F(1,16)=4.26, p=.056$. Moreover, the figure 5 showed that the electrode $\mathrm{C} 3$ was more negative than $\mathrm{C} 4$ when the cue indicated the right direction. The repeated measures ANOVA analysis in children did not show any statistical significance, in fact, they showed positive voltage values instead of the negative values showed by the young adults: right cue C3 $(M=1.06, S D=3.75)$, right cue C4 $(M=1.86, S D=4.22)$, left cue C3 $(M=1.62, S D$ $=5.15)$, left cue $\mathrm{C} 4(M=1.71, S D=4.53)$, while the young adults showed negative voltage: right cue C3 $(M=-1.12, S D=2.75)$, right cue C4 $(M=-.06, S D=2.24)$, C3 left cue $(M=-1.28, S D=$ 2.32), C4 left cue C4 $(M=-.97, S D=2.20)$ (Fig.5).

CNV in the 1560-1660 time window. At the end of the CNV period in posterior areas, it was observed a recovery of the CNV in children that did not occur in young adults. However, the mixed-model factorial ANOVA with cue, posterior areas and age group showed that there were not statistically significant effects (Fig.6).

Remaining eye movements.

Figs 2 and 3 showed the presence of remaining horizontal eye movements when the cue was directed to the right side. We think that this eye drifting can not explain the main results due to the following reasons:

-The topographical analysis of F8, F7, T8 and T7 electrodes showed some eye movements influence, following the same dynamics as the eye recordings; however, these electrodes were discarded for statistical analysis. 
- The early fronto-central negativity was accompanied by a posterior positivity (fig. 2), with a dipolar structure not compatible with eye movements. In addition, the dynamic of this component is totally different to the eye movements dynamics.

- The Posterior negativities (Fig. 3) have the foci in the posterior part of the scalp.

- The same argument is valid for the parietal positivity. Moreover, the phasic structure of this parietal component does not appear in the horizontal ocular channel.

These arguments make us confident that the observed results are due to brain activity rather than to the remaining eye movements.

\section{DISCUSSION}

The present study compared behavioral performance and ERPs in children and young adults linked with the preparatory CNV activation evoked by a warning directional cue. The preparatory activation of the specific neural resources, motor and sensory, which are going to be needed for the completion of the task, was clearly recorded in young adults, while only the sensory anticipation component was recorded in children. In addition, a parietal positivity was recorded in children suggesting a possible differential cortical engagement of brain resources in children and adults for accomplishing the task.

\section{Behavioural measures.}

Both age groups responded faster during valid trials than during invalid trials. There were not statistically significant differences among groups, although there were faster RTs in young adults with respect to children.

The increased RTs of the invalidly cued targets with respect to validly cued targets is a general result obtained in central-cue Posner's paradigm (Posner 1980). This effect has been shown in normal adults (Mangun and Hillyard, 1991, Gomez, et al, 2004 and 2008), in normal children (Enns and Brodeur, 1989, Brodeur and Boden 2000, Perchet and Garcia-Larrea, 2000, 2005) and in 
ADHD children (Perchet, 2001). The presentation of valid cues significantly decreases RTs to the incoming targets (benefits), relative to either the invalid or to the no cue and neutral conditions (costs). It has been interpreted as the consequence of the spatial attentional resources allocation in the pre-cued spatial position, producing the typical pattern of cost-benefit to invalid and valid targets (Posner, 1980, Gómez et al., 2004). In the present experiment, the invalid condition presented a higher number of omission and commission errors, indicating the difficulty of processing targets in the uncued direction. With respect to the errors, the children presented a higher number of commission errors to the $\mathrm{S} 1$ and to the $\mathrm{S} 2$ than young adults, suggesting higher impulsivity in children with respect to young adults.

\section{Event-related potential.}

Contingent Negative Variation

The early CNV component represents most likely an orienting response (Rockstroh et al., 1989). The influence of cerebral maturity in the orienting response to warning stimuli has been studied by means of the early component of the CNV. Both age groups displayed an early frontocentral negativity, but the children showed a fronto-central negativity earlier than the young adults. The children also showed a positivity posterior that coincided in latency with the fronto-central negativity. The correlation analysis and the $98 \%$ of the spatial topography variance explained by a single PCA component in the children group (Fig. 2) suggest that the fronto-central negativity and the posterior positivity are caused by a single source, not necessarily frontal. It is possible that both components, early frontal negativity and posterior positivity, were manifestations of the same component but with opposite polarities, and the fronto-central negativity does not correspond to a genuine early component of the CNV. It has been previously reported that children displayed smaller early negativity waves than adults at fronto-central locations indicating inefficient orientation processes caused by the already known immaturity of the frontal lobe (Segalowitz, et al., 1992; Jonkman, et al.,2003, Segalowitz, and Davies, 2004). At first glance, our result does not allow to confirm a reduction of the early $\mathrm{CNV}$ in children associated with the inmaturity of the 
frontal cortex, given the frontal negativity we recorded. However, Van Leeuwen et al (1998) have shown in children a quite similar topography of an early CNV frontally distributed overlapped in time with a posterior $\mathrm{P} 3$, in fact, they termed as a CNV/P3 complex. Using LORETA localization, they found posterior sources explaining both the anterior negativity and the posterior positivity. Therefore, we can assume that the anterior negativity we have obtained in children does not correspond to an early frontal negativity but to a posterior source, possibly a $\mathrm{P} 3$, propagated to anterior locations.

On the other hand, the young adults presented an early component of the CNV. The timing, morphology and topography of this early negativity corresponded closely to the early $\mathrm{CNV}$ indexing orientation to warning stimuli during preparatory processes, as it has been reported previously in the literature (Rockstroh et al., 1989, Gómez, 2001, 2003).

In midposterior areas (around $\mathrm{Pz}$ ) during the middle latencies, the children presented a positive activity that was not present in the young adults CNV (Fig.3). To our knowledge, this parietal positivity during the $\mathrm{CNV}$ has never been described previously. It is difficult to assume that it corresponds to a P300-like component given that it appears relatively late with respect to the S1 directional cue, peaking at $700 \mathrm{~ms}$ after cue. It is possible that it could represent the positive side of an extrastriate dipole explaining the posterior lateralized negativity that is discussed in next paragraph. However, as the posterior negativity remains a longer period than the parietal positivity, it is possible that the posterior negative component of the $\mathrm{CNV}$ and the parietal positivity represent different components. Although not reported, a parietal positivity can be observed in parietal sites at a similar latency in the auditory induced CNV in children (Bender, 2005). Moreover, Segalowitz et al. (2004) observed a long-lasting positivity after the cue during Go trials. If this parietal positivity is a genuine component, it would imply that children use different resources to prepare and to orient to spatial cues than young adults, probably compensating the higher immaturity of the frontal lobe in children with respect to young adults (Segalowitz et al., 2004). 
It has been proposed that the late phase of the CNV represents a motor and a sensory taskspecific pre-activation of the resources needed to complete the intended task (Gómez et al., 2001, 2003 and 2004). During adulthood, it is possible to dissociate motor and sensory attentional components related with stimulus anticipation (Brunia, 1999, Brunia and Van Boxtel, 2001, Gomez, 2003, 2004). Using auditory cues and targets, Bender (2005) found a parietal negativity that could represent the pre-activation of auditory sensory signal, but motor pre-activation was only present as an Event Related desynchronization of the sensorimotor rhythm, and it does not appear in the children CNV. In young adults, and by means of the magnetic contingent variation during the central cue Posner paradigm, the preactivation of the motor and auditory sensory cortex was obtained (Gómez et al. 2004). The data presented here conforms the presence of motor preactivation during the $\mathrm{CNV}$ in adults (only when the cue was directed to the right side), but an absence of this motor pre-activation in children (Bender et a., 2005). Moreover, the children showed positive voltage values instead of the negative values in relation to the young adults (Figs. 3 and 5).

On the other hand, a clear sensory CNV possibly originated in the visual estrastriate cortex appeared in both children and adults. It is interesting to notice the contralateral to the cue pattern of activation in the negative posterior CNV. This pattern of activation in the hemisphere contralateral to the cue over the occipital scalp gives an added support to the idea of task-specific pattern of activation in the CNV (Gómez et al., 2001, 2003 and 2004). Furthermore, the proposal of an earlier maturation of sensory cortices with respect to motor cortex is also supported in the visual modality, as it has already been proved in the auditory modality (Bender et al., 2005).

At the end of the CNV, it was possible to observe a recovery of the posterior CNV in children, although not statistically significant when compared to young adults. It is possible that the task was boring for children, decreasing the attentional control in the late part of the CNV, and initiating the recovery of the $\mathrm{CNV}$.

As a conclusion, the lack of a clear preparatory activation in motor areas and the difficulty in recording a clear early CNV in children indicates a difference between children and young adults 
preparatory processes, probably due to the influence of a lower frontal cerebral maturity in children with respect to adults. This is not the case for posterior sensory cortices which presented a quite developed CNV. Moreover, the parietal positivity in children probably suggests a different strategy to perform the task with respect to adults.

Acnowlegments. We want to thanks to Carmen Gómez-Sos for English language editorial assistance. This work was supported by the Spanish Ministry of Science and technology grant number SEJ2007/60974/pSIC and by the Junta de Anadalucia.

\section{REFERENCE}

Bender, S., Weisbrod, M., Just, U., Pfüller, U., Parzer, P., Resch, F., Oelkers-Ax, R. (2002). Lack of age-dependent development of the contingent negative variation (CNV) in migraine children? Cephalalgia, 22, 132-6.

Bender, S., Weisbrod, M., Bornfleth, H., Resch, F., Oelkers-Ax, R.(2005). How do children prepare to react? Imaging maturation of motor preparation and stimulus anticipation by late contingent negative variation. Neuroimage, 27, 737-52.

Brodeur, D.A. and Boden, C. (2000). The effects of spatial uncertainty and cue predictability on visual orienting in children. Cognitive Development, 15, 367-382.

Brunia, C.H., and Damen E.J., (1988). Distribution of slow brain potentials related to motor preparation and stimulus anticipation in a time estimation task. Electroencephalography and clinical neurophysiology, 69, 234-43.

Brunia C.H. (1999). Neural aspects of anticipatory behavior. Acta psychological, 101, 213-42.

Brunia C.H. and Van Boxtel G.J. (2001).Wait and see. International Journal of Psychophysiology, 43, 213-242. 
Chiarenza, G.A., Papakostopoulos, D., Giordana, F., Guareschi-Cazzullo, A. (1983). Movementrelated brain macropotentials during skilled performances. A developmental study. Electroencephalography and clinical neurophysiology. 56, 373-383.

Cohen, J. D., Offner, F., Palmer, C. W. (1967). Development of the continent negative variation in children. Electroencephalography and Clinical Neurophysiology, 23, 77-78.

Cohen, J. (1970). Maturation of the contingent negative variation. Electroencephalography and Clinical Neurophysiology, 28, 99.

Eimer, M. (1993). Spatial cueing, sensory gating and selective response preparation: an ERP study on visuo-spatial orienting. Electroencephalography and clinical neurophysiology, 88, $408-420$.

Enns, J.T. and Brodeur, D.A.(1989). A developmental study of covert orienting to peripheral visual cues. Journal of experimental child psychology, 48, 171-89.

Fan J., Kolster R., Ghajar J., Suh M., Knight RT., Sarkar R., McCandliss BD., (2007). Response anticipation and response conflict: an event-related potential and functional magnetic resonance imaging study. The Journal of Neuroscience, 27, 2272-2282.

Gómez, C.M, Delinte, A, Vaquero, E, Cardoso, M.J, Vázquez, M, Crommelinck, M, Roucoux, A. (2001). Current source density analysis of CNV during temporal gap paradigm. Brain Topography, $13,149-159$.

Gómez, CM., Marco, M., Grau, C. (2003). Preparatory visuo-motor cortical network of the contingent negative variation estimated by current density. Neuroimage, 20, 216-224.

Gómez, C.M., Fernandez, A., Maestu, F., Amo, C., Gonzalez-Rosa, J.J., Vaquero, E., Ortiz, T (2004). Task-specific sensory and motor preparatory activation revealed by contingent magnetic variation. Cognitive Brain Research, 21, 59-68.

Gómez CM, Flores A, Ledesma A. (2007). Fronto-parietal networks activation during the contingent negative variation period. Brain research bulletin, 73, 40-7. 
Gómez CM, Flores A, Digiacomo MR, Ledesma A, González-Rosa J. (2008). P3a and P3b components associated to the neurocognitive evaluation of invalidly cued targets.Neuroscience letters , 430, 181-185.

Hillyard, S.A. and Galambos, R. (1970). Eye movement artifact in the CNV.Electroencephalography and clinical neurophysiology, 28, 173-82.

Jonkman, L.M., Lansbergen, M., Stauder, J.E.A. (2003). Developmental differences in behavioral and event-related brain responses associated with response preparation and inhibition in a go/nogo task. Psychophysiology, 40, 752-761.

Jonkman, L.M. (2006). The development of preparation, conflict monitoring and inhibition from early childhood to young adulthood: a Go/Nogo ERP study. Brain Research, 1, 181-93.

Lee, K.M., Chang, KH., Roh, JK. (1999).Subregions within the supplementary motor area activated at different stages of movement preparation and execution. Neuroimage 9, 117-123.

Loveless, N.E. and Sanford, A.J. (1974). Slow potentials correlates of preparatory set. Biological Psychology, 1, 303-314.

Mangun, G.R. and Hillyard, S.A. (1991). Modulations of sensory-evoked brain potentials indicate changes in perceptual processing during visual-spatial priming. Journal of experimental psychology, Human perception and performance, 17, 1057-1074.

Perchet, C. and Garcia-Larrea, L. (2000). Visuo-spatial attention and motor reaction in children: an electrophysiological study of the "Posner" paradigm. Psychophysiology, 37, 231-241.

Perchet, C., Revol, O., Fourneret, P., Mauguière, F., Garcia-Larrea, L., (2001). Attention shifts and anticipatory mechanisms in hyperactive children: an ERP study using the Posner paradigm, Biological. Psychiatry, 50, 44-57.

Perchet. C. and Garcia-Larrea, L. (2005). Learning to react: anticipatory mechanisms in children and adults during a visuospatial attention task. Clinical Neurophysiology, 116, 1906-1917.

Posner, M.I. (1980). Orienting of attention. The Quarterly journal of experimental psychology. 32, $3-25$. 
Rockstroh, B., Elbert, T., Canavan, A., Lutzenberger, W., Birbaumer, N., (1989). Slow Cortical Potentials and Behavior. Urban and Schwarzenberg, Baltimore.

Van Leeuwen, T.H., Steinhausen, H.C., Overtoom, C.C., Pascual-Marqui, R.D., Van't Klooster, B., Rothenberger, A., Sergeant, J.A., Brandeis, D. (1998). The continuous performance test revisited with neuroelectric mapping: impaired orienting in children with attention deficits. Behavioral Brain Research, 94, 97-110.

Segalowitz, S. J., Unsal, A., and Dywan, J. (1992). Cleverness and wisdom in 12-year-olds: Electrophysiological evidence for late maturation of the frontal lobe. Developmental Neuropsychology, 8, 279- 298.

Segalowitz, S.J. and Davies, P.L (2004). Charting the maturation of the frontal lobe: an electrophysiological strategy. Brain and Cognition, 55, 16-33.

Yordanova, J., Dumais-Huber, C., Rothenberger, A., Woerner, W.(1997). Frontocortical activity in children with comorbidity of tic disorder and attention-deficit hyperactivity disorder. Biological Psychiatry, 41, 585- 594.

Walter, W.G., Cooper, R., Aldridge, W.J., McCallum, W.C. (1964). Contingent negative variation: an electrophysiological sign of sensoriomotor association and expectancy in the human brain. Nature, 203, 380-384.

Weerts, T.C. and Lang, P.I. (1973). The effects of eye fixation and stimulus and response location in the contingent negative variation (CNV). Biological Psychology, 1, 1-19.

Fig.1. The experimental paradigm presented two experimental conditions: a) The valid condition in which the side of target presentation is announced by a warning stimulus (cue) and it appears on the correct target side, and b) the invalid condition indeed if the target appears on the opposite side. 

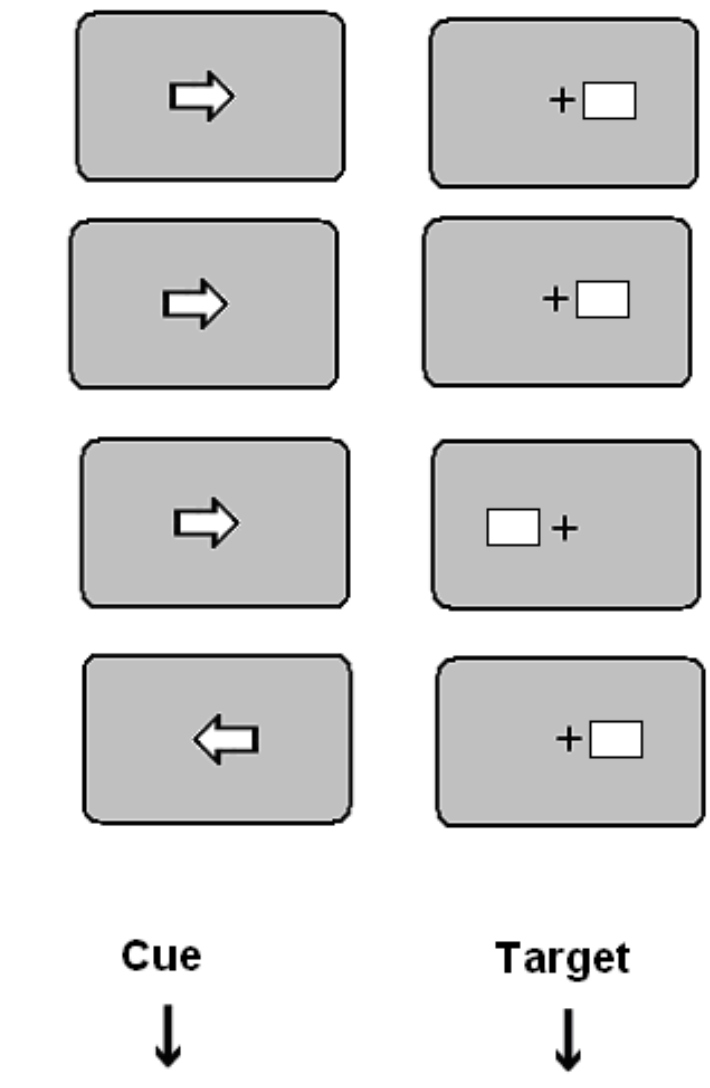

Target

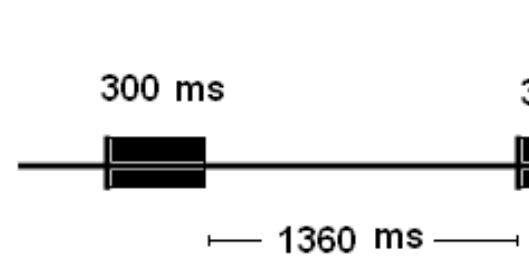

$1360 \mathrm{~ms}$

\section{Valid condition}

$(82.1 \%)$

\section{Invalid \\ condition}

$(17.9 \%)$

Fig.2 Grand average ERPs and topography in response to Cues (left and right collapsed) in children and young adults. The long vertical lines crossing all the channels correspond to the arrival time of the cue (S1) and the target (S2) respectively. The time period between these two lines corresponds to the period in which the CNV develops. The two short parallel lines correspond to the time windows in which the early $\mathrm{CNV}$ is analyzed and the topographical maps are displayed, these time windows are $330-450 \mathrm{~ms}$ for young children and $450-550 \mathrm{~ms}$ for young adults. a) Notice that the fronto-central negativity in children follows the same dynamics that a positive component in posterior sites, b) The children showed a fronto-central negativity earlier than the young adults. The voltage topographical map is displayed (second map from the left) and it follows a very similar 
topography than the map for the first principal component (first map from the left) (see details in the text). The map in the right below corresponds to the early fronto-central negativity in young adults that could correspond to the early $\mathrm{CNV}$. The $\mathrm{H} 1-\mathrm{H} 2$ recording corresponds to horizontal electroculogram.

a)

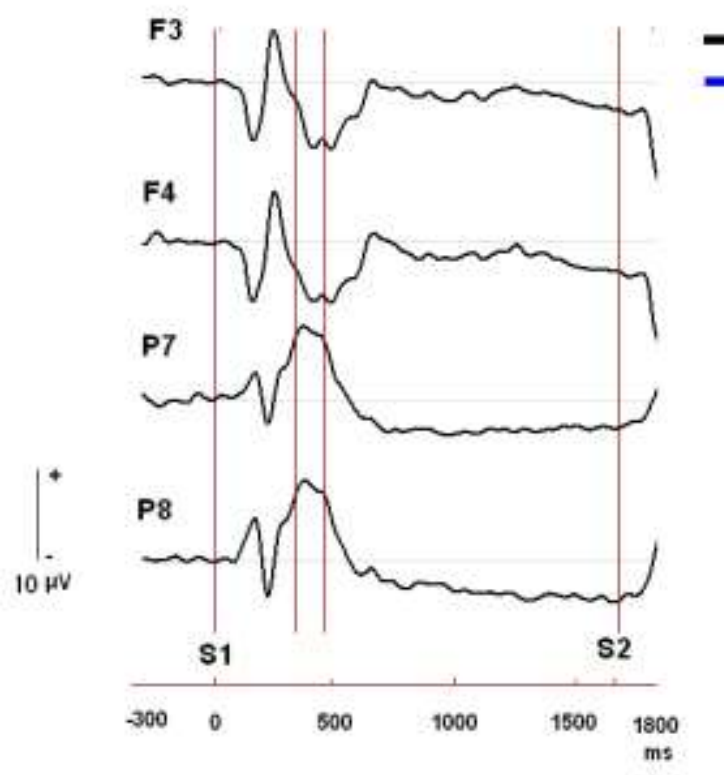

b)

Children

Young adults

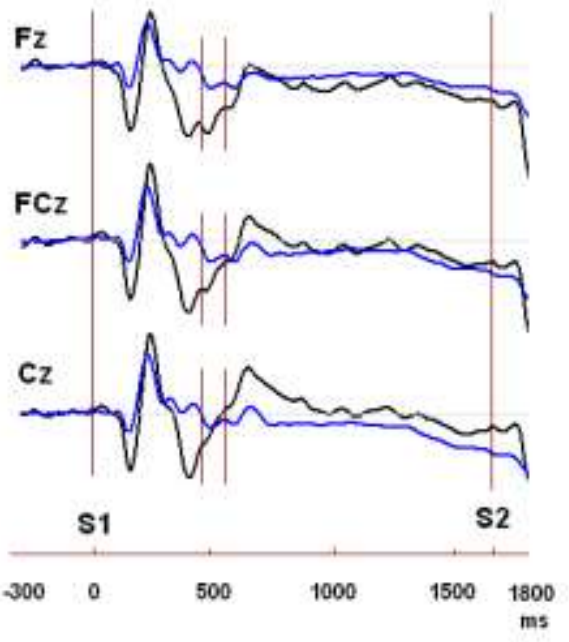

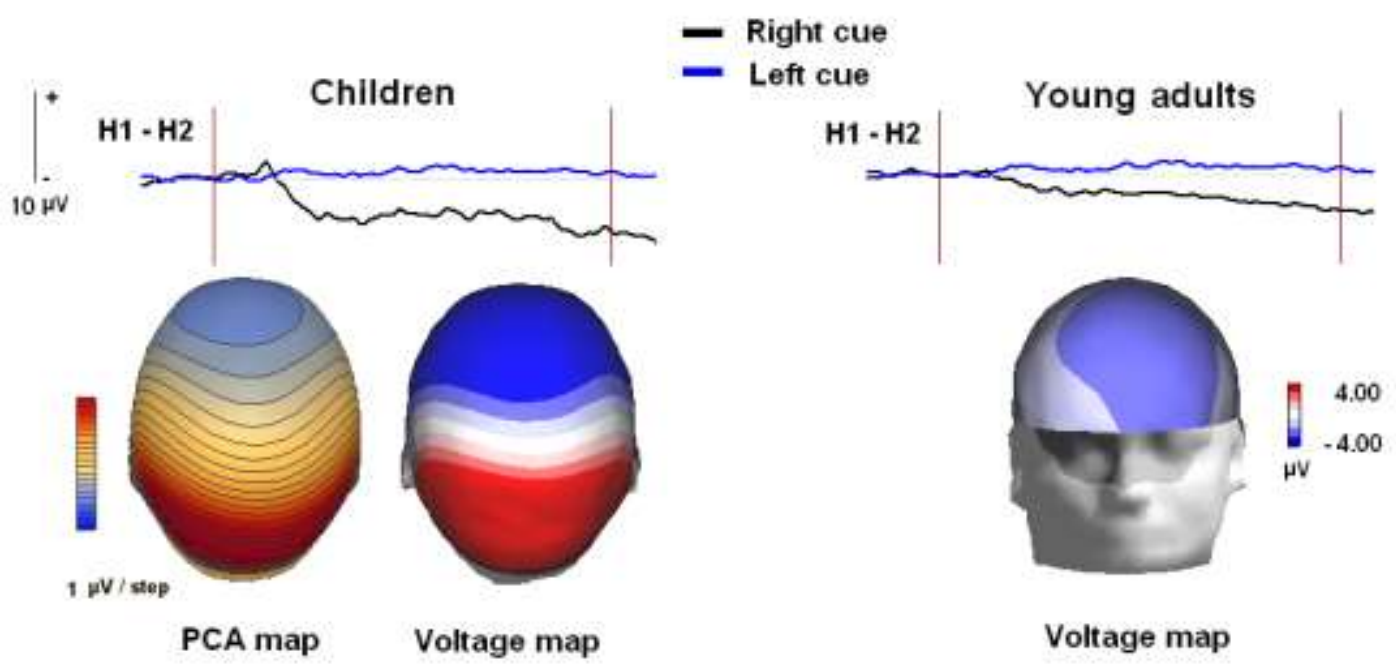

Fig.3 Grand average ERPs and topography in response to left cues and right cues in children and young adults. C3-C4 and $\mathrm{O} 1-\mathrm{O} 2$ correspond to the substraction of these electrodes. The 
topographical maps correspond to the $\mathrm{CNV}$ intermediate latency $(550-950 \mathrm{~ms})$ marked in the figure with two parallel segments. Notice that in children a parietal positivity appears, which is absent in the young adults. Moreover, in both age groups, a negativity contralateral to the cue appeared in posterior sites. The $\mathrm{H} 1-\mathrm{H} 2$ recording corresponds to horizontal electroculogram.
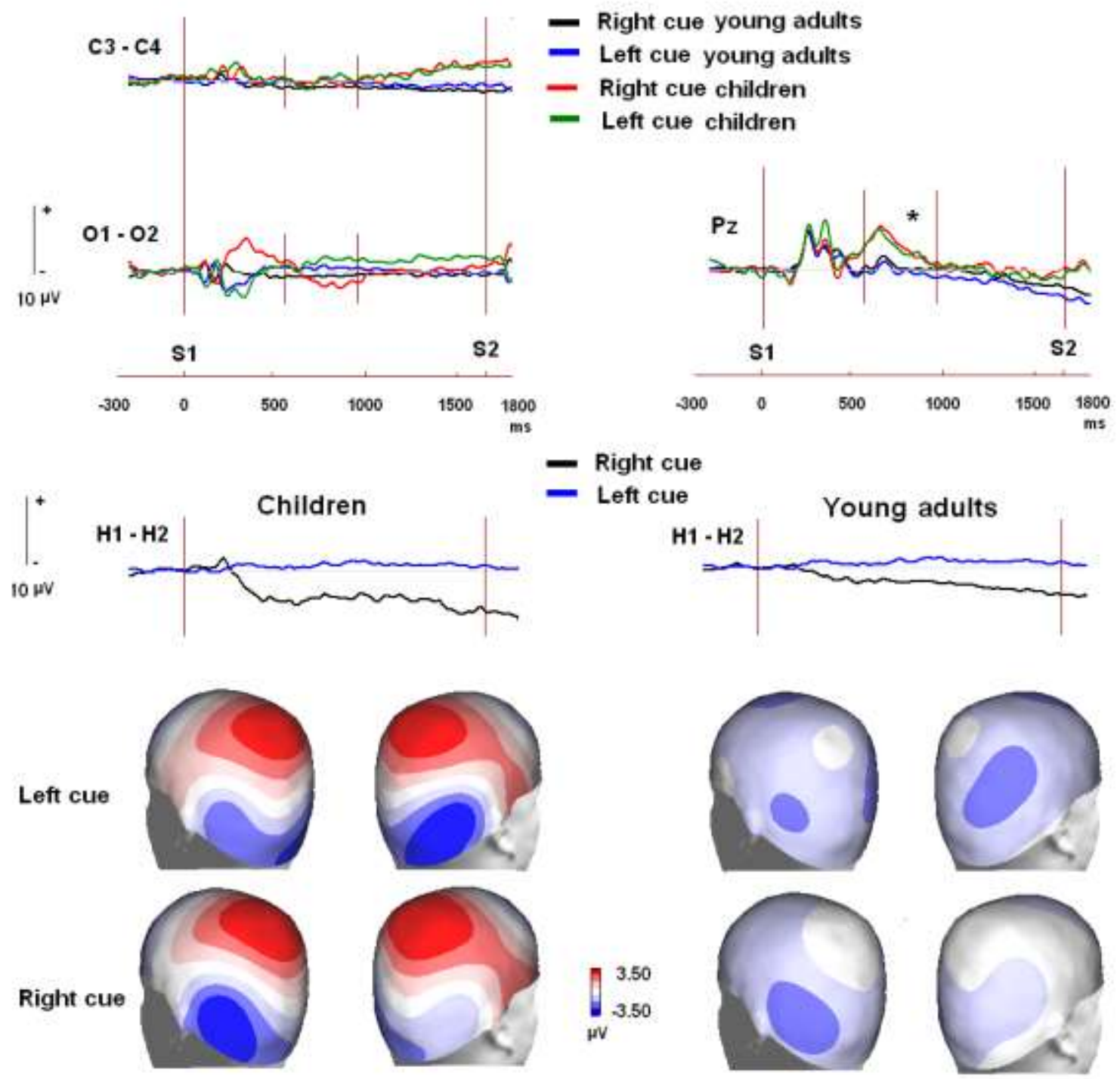

Fig.4. Voltage values of the effects of factors interactions interaction during the time window of 550 and $950 \mathrm{~ms}:$ a) the interaction between the effects of age group and posterior areas, and b) the effects of the interaction between the cue and posterior area factors. The analyzed areas were leftposterior (collapsing P3, P7, O1), midposterior (Pz) and rigt-posterior (collapsing P4, P8, O2). 
a)

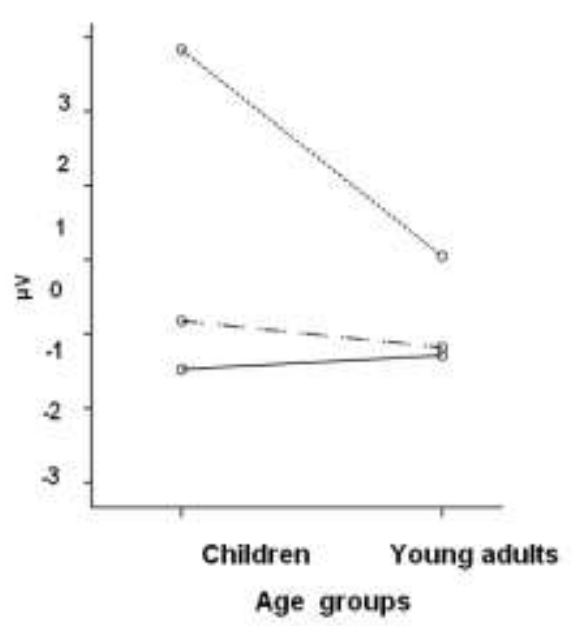

b)

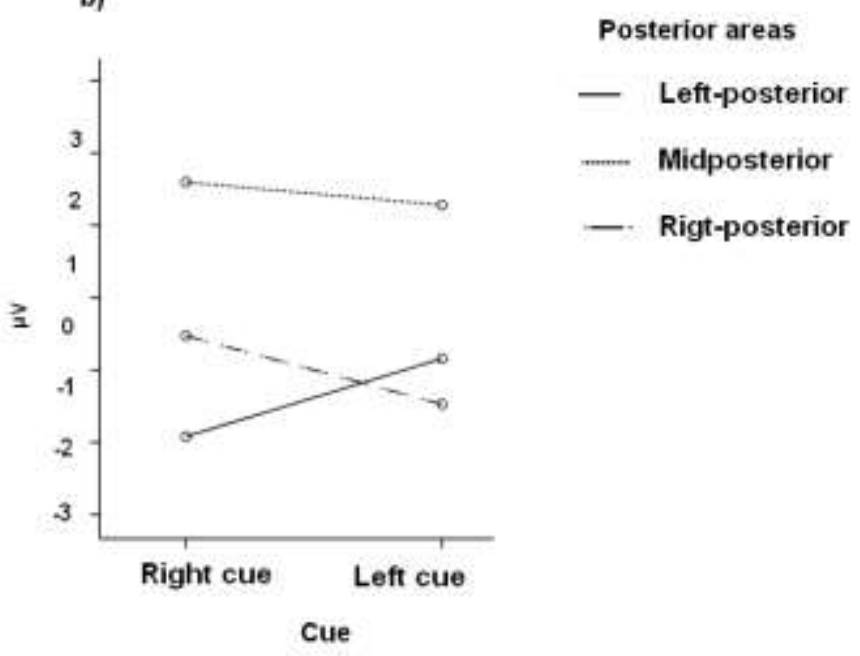

Fig.5. Voltage values of the electrodes $\mathrm{C} 3$ and $\mathrm{C} 4$ when left and right cues are presented during the time window of 550 and $950 \mathrm{~ms}$. Notice that while the voltage values are negative in young adults, those values are positive in children. Also notice the hemispheric difference (more negative in C3 than in $\mathrm{C} 4$ ) in young adults during the right cue condition.

Children

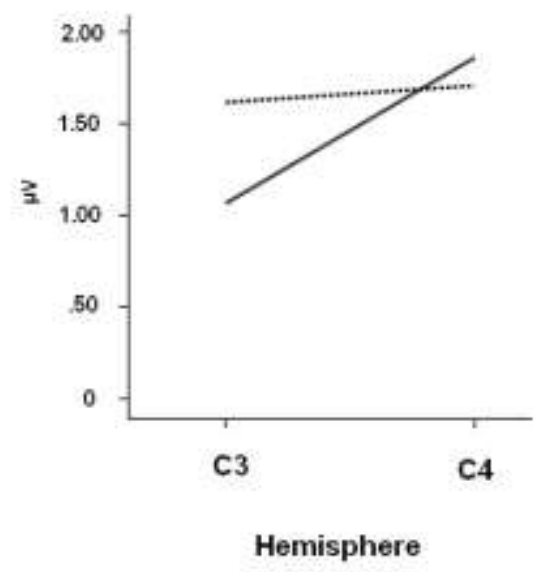

Young adults

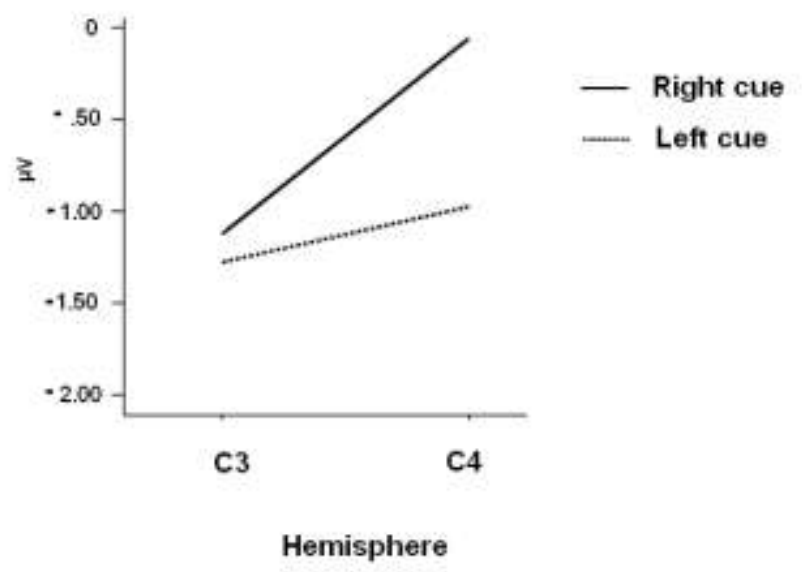

Fig.6. Topographical map for the late CNV (1560-1660). Notice that most of the scalp is negative in young adults. However, an early CNV recovery (not statistically significant) appeared in posterior sites in children. 

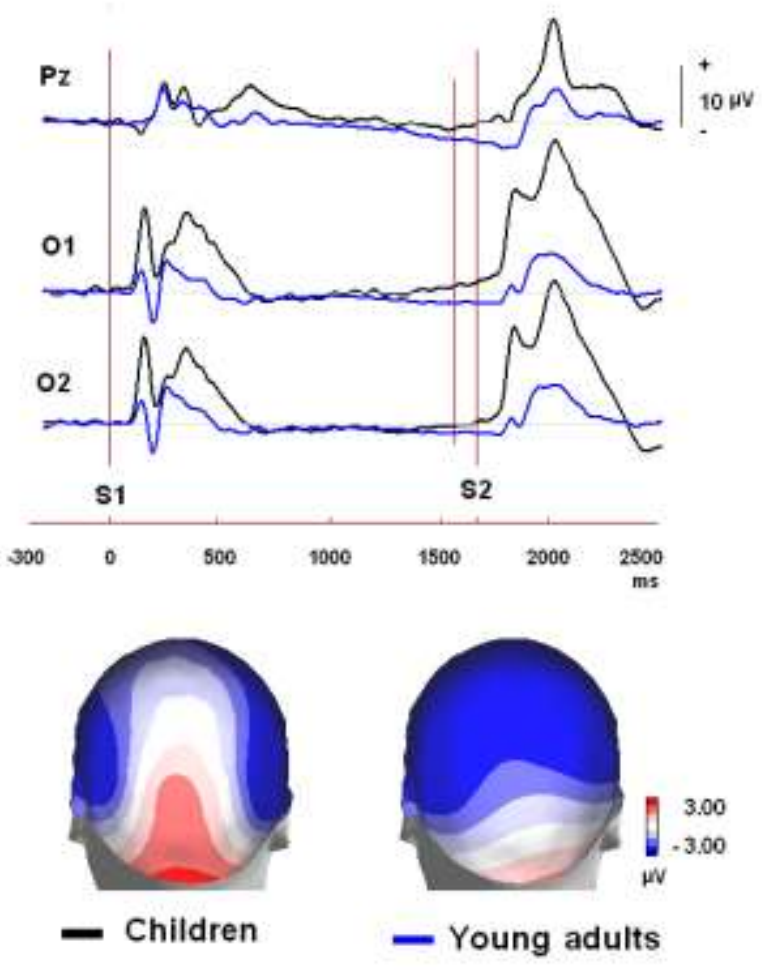

Table 1. Reaction times of the four experimental stimular sequences analyzed.

\begin{tabular}{llcc}
\hline Condition & Groups & Reaction Times & S.D \\
\hline Valid cue & Children & 394.34 & 74.89 \\
& Young adults & 343.90 & 87.11 \\
& & & \\
Invalid cue & Children & 423.84 & 69.80 \\
& Young adults & 387.75 & 91.50 \\
\hline
\end{tabular}

Table 2. Percentages of errors in the valid and invalid conditions are shown. The types of errors considered were percentage of no response (Omissions to the S2), percentage of false alarms to the S1 (Responses to the S1) and percentage of response errors to the target stimuli (commission errors). 


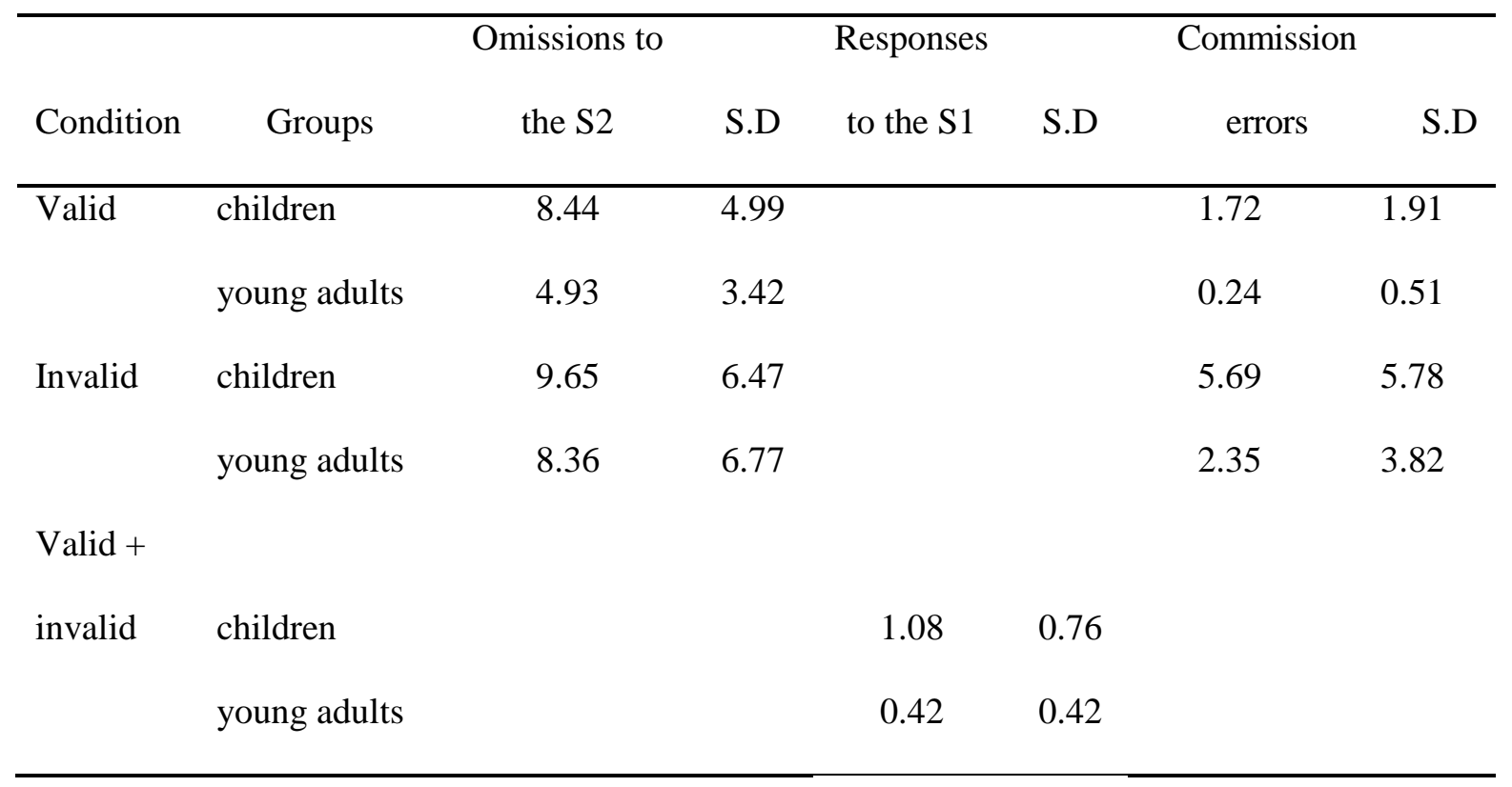

